\title{
A Novel Curvelet Thresholding Function for Additive Gaussian Noise Removal
}

\author{
Yaser Norouzzadeh and Mahdi Jampour
}

\begin{abstract}
Improving quality of noisy images has been an active area of research in many years. It has been shown that removing additive Gaussian noise by nonlinear methods such as Wavelet denoising and Curvelet denoising had better results than classic approaches. However estimation of threshold and selection of thresholding function are still challenging tasks. In this paper, a new thresholding function is proposed for Curvelet thresholding and thresholding neural network is extended to use Curvelet coefficients instead of wavelet coefficients. This function is continues and has higher order derivation. Therefore it is suitable for gradient decent learning methods such as thresholding neural network (TNN). This function is used by the TNN and threshold values for Curvelet sub-bands are estimated according to least mean square (LMS) algorithm. The experimental results show improvement in noise reduction from images based on visual assessments and PSNR comparing with well-known thresholding functions.
\end{abstract}

Index Terms-Image denoising, curvelet thresholding, Thresholding function, thresholding neural network.

\section{INTRODUCTION}

Images may be corrupted by noise in acquisition and transmission phases. Various noise removal methods reported by researchers. Linear methods have some side effects while removing noises. Therefore non-linear denoising methods in wavelet domain have been an active area for two decades. It is interested for researchers due to its ability to improve quality of noisy images.

Wavelet domain based noise removal techniques need some threshold value to removing small coefficients of detail sub-bands and preserving large coefficients; because small coefficients are usually noisy and large coefficients contains main features of image. Thresholding needs a thresholding function to decide how improve coefficients by using threshold. Therefore, estimating threshold and determining thresholding rules are still challenging problems in wavelet denoising.

The methods for estimation of threshold are divided in three groups. First group uses universal threshold value for all wavelet sub bands of noisy image [1], [2]. In second group, namely sub band-adaptive, the thresholds are determined differently for any detail sub band [3]-[9]. Spatially adaptive thresholds are selected for each wavelet coefficient or some group of them in third group [10].

Manuscript received February 9, 2011; revised July 10, 2011.

Yaser Norouzzadeh is with Computer Engineering Department at Shahid Bahonar University of Kerman Kerman, Iran (email: YNR@Mail.uk.ac.ir)

Mahdi Jampour is with Computer and IT Department at Kerman Institute of Higher Education (KIHE) Kerman, Iran (email: Jampour@ieee.org)
Hard and soft thresholding functions [1] are the most commonly used thresholding functions. Hard thresholding is discontinues and is not differentiable. Soft thresholding is continues but does not have first order derivation. Therefore these thresholding functions can't be used in gradient based learning tools such as Thresholding Neural Networks [11], [12]. Some New thresholding functions such as garrote [13] and Zhang functions [12], [14] are reported in recent years. Garrote thresholding function has better properties than hard and soft thresholding, but they are not differentiable.

In recent years Curve let transform has become more interested in image processing tasks such as image denoising. In this paper, concept of TNN in extended to use Curve let coefficients instead of wavelet coefficients. In addition a new thresholding function is proposed which is continues and differentiable. Hence it has higher order derivation and can be used by TNN. So, the proposed thresholding function can be tuned using LMS algorithm in TNN. In other words, in the learning process the best threshold value is obtained for the proposed thresholding function. This paper includes following sections. Section 2 explains the existing wavelet denoising methods in brief. In section 3 Curve let transform is described. Some background of TNN is reviewed in section 4. Section 5 describes the proposed thresholding function. Section 6 represents comparison of the proposed method with the most well- known image denoising methodologies. Finally section 7 concludes the paper.

\section{WAVELET DENOISING}

Let the image is defined by $f(i, j), i, j=1,2, \ldots, N$ where $N$ is an integer power of 2 . If $f(i, j)$ is corrupted with additive white Gaussian noise $n(i, j)$, the observed noisy image $g(i, j)$ will be given by (1):

$$
g(i, j)=f(i, j)+n(i, j) \quad i, j=1,2, \ldots, N
$$

The goal of image denoising is to remove the noise from $g(i, j)$ and estimate $\hat{f}(i, j)$ which minimizes the mean square error (MSE):

$$
M S E=\frac{1}{N^{2}} \sum_{i=1}^{N} \sum_{j=1}^{N}[\hat{f}(i, j)-f(i, j)]^{2}
$$

In the recent years, there has been a large amount of research on the image denoising based on WT [15]. In wavelet domain, small wavelet coefficients are more likely to be noise, while large coefficients are major feature of original image. To decide which coefficient is small, a threshold is 
needed. Estimation of threshold is a major problem in this filed. One of the first methods for estimation of threshold was VisuShrink [1]. In this method the value of threshold is obtained from $\sigma \sqrt{2 \log L}$. Here $\sigma$ is noise variance and $L=N^{2}$ is the size of image. Sure Shrink [1] which is based on minimizing the Stein's unbiased risk [5] has better results than VisuShrink.

The most popular thresholding methods are soft and hard thresholding. They are given by (3) and (4), respectively.

Soft thresholding is a continuous function while hard thresholding is a discontinuous function which causes some artifacts in denoised image. Therefore soft thresholding method is preferred on hard thresholding [1], [16].

$$
y_{\text {Soft }}(t)= \begin{cases}\operatorname{sgn}(x(t)) \cdot(|x(t)-t h r|) & |x(t)|>t h r \\ 0 & |x(t)|<t h r\end{cases}
$$

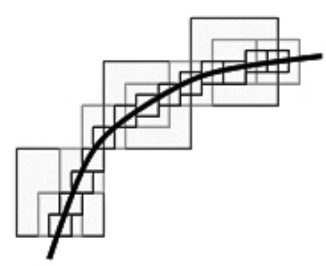

Fig. 1. Wavelet (left) versus Curve let (right). Curve let can represent curves better than Wavelet

\section{CURVE LET TRANSFORM}

Curve let transform theory is introduced in recent years and it is under development [17]-[19]. Major advantages of Curve let are: Directionality and Anisotropy; Wavelet allows us to analysis image in three different directions (Vertical, horizontal and Diagonal), but Curve let support more directions. To capture smooth curves, basis element should use a variety of shapes with different aspect ratios which cause Curve let have Anisotropy [18]. Therefore Curve let transform is more powerful than Wavelet to represent curves as is shown in Fig. 1. Thus Curve let have better results in image denoising than wavelet [17]. In [20] the Curve let transform based on Ridge let transform is described. The Continues Ridge let Transform (CRT) represents smooth functions and straight edges sparsely. The CRT for two dimensional functions $f\left(x_{1}, x_{2}\right)$ has following form:

$$
\varphi_{(a, b, \theta)}\left(x_{1}, x_{2}\right)=a^{-1 / 2} \varphi\left(\frac{x_{1} \cos \theta+x_{2} \sin \theta-b}{a}\right)
$$

Mentioned CRT can be calculated using Wavelet in a domain which defined by (8):

$$
R_{f}(\theta, t)=\int_{R^{2}} f\left(x_{1}, x_{2}\right) \delta\left(x_{1} \cos \theta+x_{2} \sin \theta-t\right) d x_{1} d x_{2}
$$

Where $(\theta, t) \in[0,2 \pi) \times R$ and $\delta$ is Dirac distribution. Thus by applying one dimensional wavelet transform to $R_{f}(\theta, t)$, the CRT is obtaining [21]. Equation (9) shows this relation:

$$
C R T_{f}(a, b, \theta)=a^{-1 / 2} \int_{R} \varphi\left(\frac{t-b}{a}\right) R_{f}(\theta, t) d t
$$

In image processing, edges are curves rather than straight

$$
y_{\text {Hard }}(t)= \begin{cases}x(t), & |x(t)|>t h r \\ 0, & |x(t)|<t h r\end{cases}
$$

Where $t h r$ is threshold and $y$ and $x$ are modified and noisy version of image in the wavelet domain, respectively.

Zhang's thresholding functions (5) and (6) are continues and differentiable. Hence they don't have disadvantages of soft and hard functions. As a result they are suitable for use in the TNN.

$$
f(x, t h r, k)= \begin{cases}x+t h r-\frac{t h r}{2 k+1} & x<-t h r \\ \frac{1}{(2 k+1) t h r^{2 k}} x^{2 k+1} & |x| \leq t h r \\ x-t h r+\frac{t h r}{2 k+1} & x>t h r\end{cases}
$$

In (5) and (6), $x$ is wavelet coefficients of noisy image, thr is threshold value, $k$ and $\lambda$ are parameters for adjusting the shape of thresholding functions.

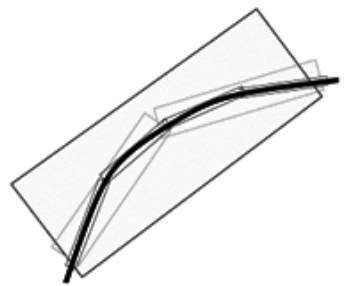

lines. So Ridge let alone isn't effective to represent edges. Although Curve lets are based on Ridge let, but Curve let can separates different scales using band-pass filtering in special domain [22, 23]. Curve lets occur in all scales, directions and locations as Ridge let. Ridge lets have global lengths and variable widths, while Curve lets have variable lengths and widths.

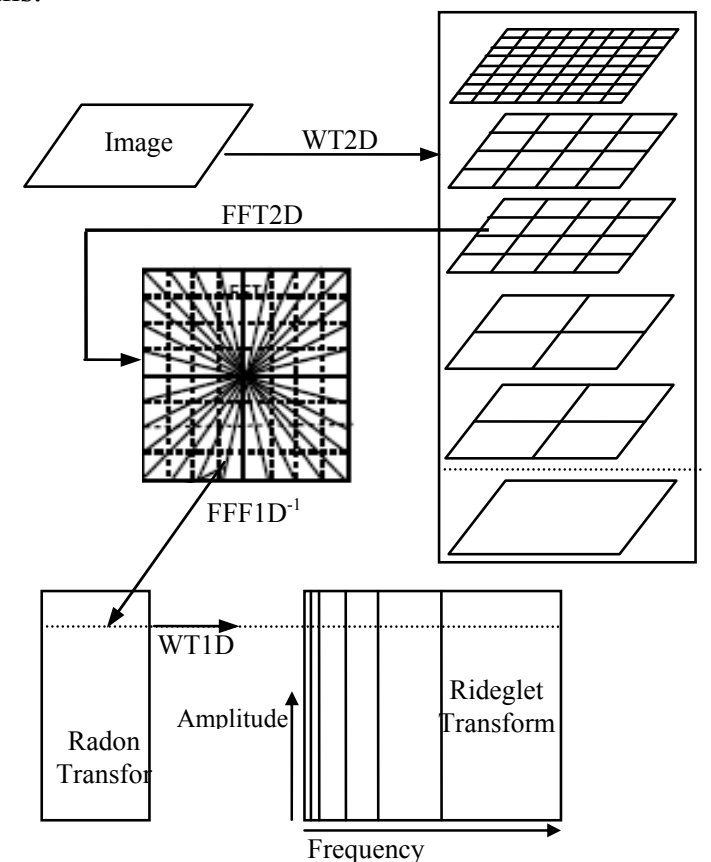

Fig. 2. Curve let transform flow graph. The figure illustrates the decomposition of the original image into sub bands followed by the spatial partitioning of each sub band. The Ridge let transform is then applied to each block.

Discrete Curve let transform using scale and band-pass 
filter banks $\left(P_{0} f, \Delta_{1} f, \Delta_{2} f, \ldots\right)$ where band-pass filter $\Delta_{s}$ is near of $\left[2^{2 s}, 2^{2 S+2}\right]$ frequencies. The Curve let transform steps are illustrated in Fig. 2.

In this work, real version of Fast Discrete Curve let Transform (FDCT) [19] is used. FDCT is based on regular rectangular grid instead of tiled grid. Here, number of directions and levels are 16 and 6, respectively.

\section{THRESHOLDING NEURAL NETWORK}

Thresholding Neural Network is combination of two concepts: neural network and wavelet thresholding [12]. In TNN, thresholding function is used instead of activation functions in feed forward neural network and TNN weights are constant and equal to 1 . Consequently threshold value can be adjusted in learning phase. In other words in neural network activation function structure is constant and weights are changing in learning process but in TTN weights are constant and thresholding function structure can be tuned by threshold value. Fig. 3 represents TNN structure. Inputs of TTN are wavelet coefficients $\left(u_{i}\right)$ of noisy image $(y)$ and outputs are thresholded wavelet coefficients $\left(v_{i}\right)$. After inverse wavelet transform denoised image is available $(\hat{x})$. In this paper, wavelet coefficients replaced by Curve let coefficients.

TNN learning method is least mean squares (LMS). In step $\mathrm{j}$ of learning, threshold value is adjusted using (10):

$$
\operatorname{thr}(j+1)=\operatorname{thr}(j)+\Delta \operatorname{thr}(j)
$$

where $\Delta t h r(j)$ is calculated by $(11)$ :

$$
\Delta \operatorname{thr}(j)=-\left.\alpha \frac{\partial M S E}{\partial t h r}\right|_{t h r=t h r(j)}
$$

where $\alpha$ is learning rate.

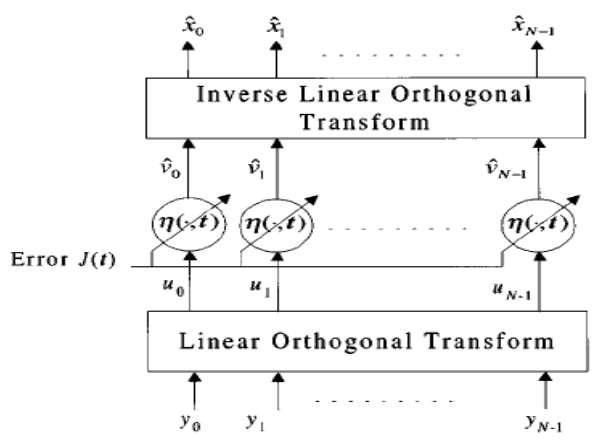

Fig. 3. Zhang Thresholding neural network structure [12]

\section{Proposed Thresholding FunCTION}

Equation (12) shows proposed thresholding function:

$$
f(x, t h r)=x-\frac{x}{\exp \left[(x / t h r)^{2}-1\right]}+\frac{1 / 8 x}{\exp (x / 0 / 71 t h r)^{2}}
$$

Where $x$ is noisy image and thr is threshold value. This function is continues, differentiated and have higher order derivation. Hence it is suitable for TNN and any gradient decent learning algorithm. Fig. 4 shows proposed thresholding function for $t h r=3$. Comparison of proposed thresholding function with hard thresholding function and soft thresholding function is shown in Fig. 5. As can be seen thresholding function is near to zero in $[-t h r, t h r]$ and for other threshold values it converges to $f(x, t h r)=x$. Therefore noisy coefficients in $[-t h r, t h r]$ are shrieked. In addition proposed thresholding function is one to one near of thr, while Zhang thresholding functions don't have this property.

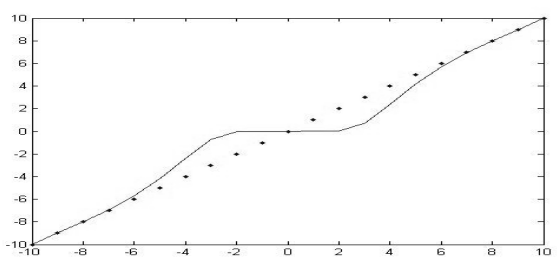

Fig. 4. Proposed thresholding function (12) with $t h r=3$ (dark line) compared with $f(x)=x$ (dotted line)

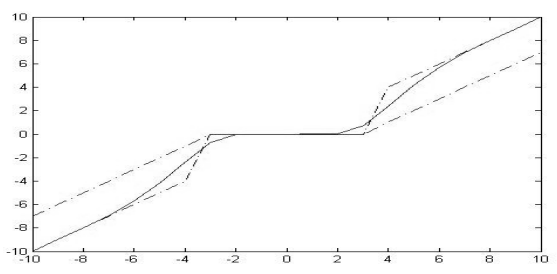

Fig. 5. Comparing proposed thresholding function (12) (dark line) with soft thresholding and hard thresholding(dotted line)

Also proposed thresholding function doesn't need any parameters except threshold value. Therefore it can be adjusted easily.

Equations (13) and (14) represent other proposed thresholding functions. They are continues and they have high order derivation, but shape of these thresholding functions is not as good as first proposed thresholding function. Also they have more parameters which make tuning of functions more difficult. Fig. 6 and Fig. 7 represent these functions for $t h r=3$.

$$
\begin{gathered}
f(x, a)=x-\frac{a^{2} x}{x^{8}+a^{2}} \quad ; a=1500 t h r \\
f(x, a)=x-\frac{a^{2} x}{x^{2}+a^{2}} ; a=1 / 5 \text { thr }
\end{gathered}
$$

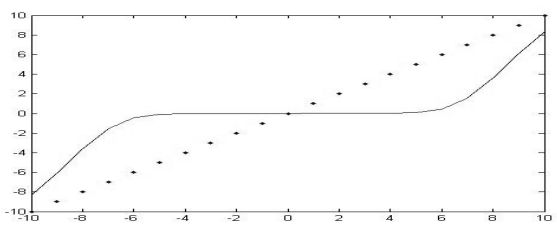

Fig. 6. Second proposed thresholding function (13) with $t h r=3$ (dark line) compared with $f(x)=x$ (dotted line)

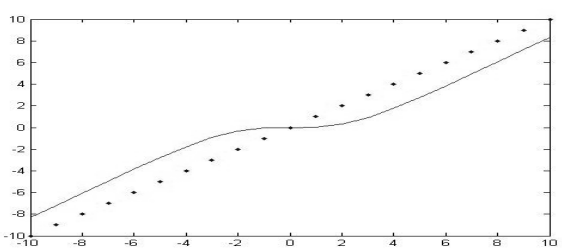

Fig. 7. Second proposed thresholding function (14) with $t h r=3$ (dark line) compared with $f(x)=x$ (dotted line) 


\section{EXPERIMENTAL RESULTS}

Proposed thresholding function is used in TTN. The $256 \times 256$ "Lena" image is used in training phase for each noise variance. First Gaussian noise is added to this image and then FDCT of noisy image is computed to provide TNN inputs. Learning rate, convergence value are chosen 1e-6 and $1 \mathrm{e}-6$, respectively and universal threshold value is obtained during learning process. TNN uses proposed thresholding function (6). In test phase, computed threshold value in learning phase is used by proposed thresholding function to demised Curve let coefficients of test images. Fig. 8 shows this process. Table 1 shows experimental results for various thresholding functions. It can be seen proposed thresholding function has produced better results in terms of Peak-to-Signal-Noise-Ratio (PSNR) value.

\section{CONCLUSION}

In this paper an effective thresholding function is proposed which utilize TNN for tuning universal threshold value. This function is continues and have higher order derivation which make it suitable for gradient decent learning algorithms such as TNN. In addition proposed thresholding function doesn't need to additional parameters. Therefore problem of tuning parameters is resolved. In previous works, wavelet coefficients are used by TNN, but in this work Curve let coefficients is used. Using sub-band adaptive threshold value in TNN can be used in future works.

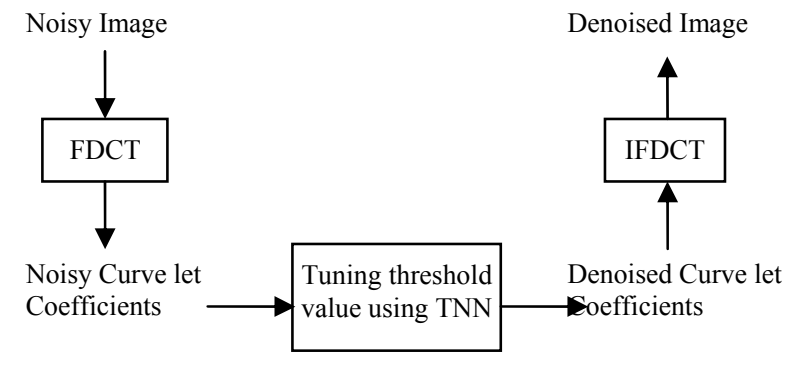

Fig. 8. Block diagram of thresholding neural network using proposed thresholding function (12).

TABLE 1: COMPARING Proposed Thresholding Function With OTHER Thresholding Functions On PSNR VALUE

\begin{tabular}{|c|c|c|c|c|c|c|}
\hline Image & $\sigma$ & Soft & Hard & Garrote & Zhang 01 & $\begin{array}{l}\text { Proposed Thresholding } \\
\text { function (12) }\end{array}$ \\
\hline \multirow[t]{3}{*}{ Lena } & 10 & 24.89 & 27.95 & 26.22 & 29.91 & 32.05 \\
\hline & 20 & 22.66 & 25.04 & 23.57 & 25.74 & 28.16 \\
\hline & 30 & 21.61 & 23.48 & 22.27 & 23.92 & 26.05 \\
\hline \multirow[t]{3}{*}{ Barbara } & 10 & 22.18 & 24.83 & 23.23 & 28.97 & 30.25 \\
\hline & 20 & 20.38 & 22.06 & 21.03 & 24.05 & 25.82 \\
\hline & 30 & 19.55 & 20.92 & 20.05 & 21.83 & 23.69 \\
\hline \multirow[t]{3}{*}{ Cameraman } & 10 & 21.76 & 24.81 & 23.08 & 28.85 & 30.05 \\
\hline & 20 & 19.49 & 21.79 & 20.43 & 23.8 & 25.58 \\
\hline & 30 & 18.39 & 20.17 & 18.97 & 21.38 & 23.16 \\
\hline
\end{tabular}

\section{REFERENCES}

[1] D.L. Donoho, I.M. Johnstone, "Ideal spatial adaptation by wavelet shrinkage", Biometrika 81 (3), 1994, pp. 425-455.

[2] M. Eftekhari, Y. Norouzzadeh and S.D. Katebi, "Wavelet denoising using genetic programming". 7th Conference of Intelligence Systems -, Tehran, Iran, 2005, (In Persian).

[3] S. Chang, B. Yu, M. Vetterli, "Adaptive wavelet thresholding for image denoising and compression”, IEEE Trans. Image Process. 9, 2000, 1532-1546.

[4] M.S. Crouse, R.D. Nowak, R.G. Baraniuk, "Wavelet-based signal processing sing hidden Markov models", IEEE Trans. Signal Process. 46, 1998, 886-902.

[5] D.L. Donoho, I.M. Johnstone, "Adapting to unknown smoothness via wavelet shrinkage", J. Am. Stat. Assoc. 90 (432), 1995, 1200-1224.

[6] Y. Norouzzadeh, S.D Katebi, "Application of ANFIS in Wavelet Denoising". 6th Iranian Conference on Fuzzy Systems and 1th Islamic World Conference on Fuzzy Systems، Islamic Azad University of Shiraz، 2006. pp. 570.

[7] Y. Norouzzadeh, S.D Katebi،"Threshold estimation in wavelet domain using genetic programming", 4th Iranian Conference on Machine Vision and Image Processing, 2007.

[8] Y. Norouzzadeh, S.D Katebi,"Threshold estimation in wavelet domain using fuzzy rules", 4th Iranian Conference on Machine Vision and Image Processing, 2007. (In Persian).

[9] Y. Norouzzadeh, S.Z Azimi far, "Curvelet denoising using neural network", 12th Annaul International CSI Computer Conference, 2007, pp. 1846-1851, (In Persian).

[10] M.K. Mihcak, I. Kozintsev, K. Ramchandran, P. Moulin, "Low-complexity image denoising based on statistical modeling of wavelet coefficients", IEEE Signal Process. Lett. 6, 1999, 300-303.

[11] X.-P. Zhang, "Space-scale adaptive noise reduction in images based on hresholding neural networks", in: Proceedings of IEEE International
Conference on Acoustics, Speech, and Signal Processing, 2001, pp. 1889-1892.

[12] X.-P. Zhang, "Thresholding neural network for adaptive noise reduction", IEEE Trans. Neural Networks 12 (3), 2001, 567.

[13] H. Gao, "Wavelet shrinkage denoising using the nonnegative garrote", J. Compute. Graph. Stat. 7, 1998, 469-488.

[14] X.-P. Zhang, M.D. Desai, "Adaptive denoising based on SURE risk", IEEE Signal Process. Lett. 5 (10), 1998, 265

[15] A. Antoniadis, J. Bigot and T. Sapatinas, "Wavelet estimators in nonparametric regression: a comparative simulation study". Journal of Statistical Software, Vol. 6, Issue 6, 2001, 1-83.

[16] R. A. DeVore and B. J. Lucier, "Fast wavelet techniques for near-optimal image processing", in IEEE Military Communications Conf. Rec. San Diego, Oct. 11-14, 1992, vol. 3, pp. 1129-1135.

[17] Starck, J.L., Candes, E., and Donoho, D.L., "The Curve let Transform for Image Denoising", IEEE Transactions on Image Processing , 11, 6, 2002, pp $670-684$.

[18] Candes, E. J., and Donoho, D. L., "New tight frames of curve lets and optimal representations of objects with piecewise-C2 singularities", Comm. on Pure and Appl. Math. 57, 2004, 219-266.

[19] Candes, E. J., Demanet, L., Donoho, D. L., and Ying, L., "Fast discrete curvelet transforms", tech. rep., Applied and Computational Mathematics, California Institute of Technology, 2005.

[20] Do, M.N., and Vetterli, M., "The finite ridgelet transform for image representation", IEEE trans. on Image Processing, vol. 12, no 1, 2002, pp. 16-28.

[21] Campisi, P., Kundur, D. and Neri, A., "Robust Digital Watermarking in the Ridgelet Domain", IEEE Signal Processing Letters, vol. 11, no. 10, 2004, pp. 826-830.

[22] Donoho, D.L., and Duncan, M.R., "Digital Curve let transform: Strategy, implementation and experiments", Proc. SPIE, vol. 4056, 2000, pp. 12-29.

[23] Jampour, M. Ziari, M. Zadeh, R.E. and Ashourzadeh, M. "Impulse noise detection and reduction using fuzzy logic and median heuristic filter" Int. Conf. Networking and Information Technology (ICNIT), Manila, 2010, pp: 19-24. 